\title{
IL-27, targeting antigen-presenting cells, promotes Th17 differentiation and colitis in mice
}

\author{
A Visperas ${ }^{1,2}$, JS Do ${ }^{1}, \mathrm{~K}$ Bulek ${ }^{1}, \mathrm{X} \mathrm{Li}^{1}$ and B Min ${ }^{1,2}$
}

T helper type 17 (Th17) cells have been implicated in autoimmunity and inflammatory bowel disease (IBD). Antigen-presenting cell (APC) -derived cytokines such as interleukin (IL)-1ß and IL-6 are key mediators supporting Th17 differentiation, yet how these factors are induced in vivo remains unclear. Here, we show that IL-27 acting on APCs enhances IL-6 and IL-1 $\beta$ production and Th17 differentiation. IL-27R $\alpha-/$ - T-cell receptor (TCR) $\beta-/$ - recipients fail to develop gut inflammation following naive CD4 T-cell transfer, whereas IL-27R $\alpha+/+$ TCR $\beta-/-$ recipients develop severe colitis. Investigation of T-cell responses exhibits that IL-27R $\alpha-/$ - TCR $\beta-/$ - mice do not support Th17 differentiation with significantly decreased levels of IL-6 and IL-1 $\beta$ by APCs. Our study has identified a novel proinflammatory role for IL-27 in vivo that promotes Th17 differentiation by inducing Th17-supporting cytokines in APCs.

\section{INTRODUCTION}

Upon antigen stimulation, naive CD4 T cells differentiate into distinct effector T-cell subsets. ${ }^{1}$ One key determinant involved in this process is cytokines primarily produced by non- $\mathrm{T}$ cells, mainly antigen-presenting cell (APCs). Interleukin (IL)-12 produced by activated APCs promotes naive T-cell differentiation into interferon $\gamma$ (IFN $\gamma$ )-producing Th1 (T helper type 1) cells via signal transducer and activator of transcription 4 (STAT4) and T-bet. ${ }^{1}$ Combination of IL-1 $\beta$, IL-6, IL-23, and tumor growth factor- $\beta$ (TGF $\beta$ ) drives Th17 differentiation via STAT3 and ROR $\gamma$ t (retinoid-related orphan receptor $\gamma){ }^{1}$ Dysregulated proinflammatory responses have been linked to inflammatory disorders including inflammatory bowel disease (IBD) and multiple sclerosis. ${ }^{2,3}$ In a mouse model of IBD, naive CD4 $\mathrm{T}$ cells transferred into immunodeficient hosts differentiate into effector cells producing Th1 and Th17 cytokines and induce chronic inflammation in the colon. ${ }^{4} \mathrm{~A}$ cellular mechanism(s) underlying the induction and regulation of Th17-type inflammatory immunity still remains unclear.

IL-27 is a heterodimeric cytokine consisting of the subunits IL-27p28 and Epstein-Barr virus-induced gene 3 (Ebi3), produced by activated APCs. It binds to the IL-27 receptor consisting of IL-27R $\alpha$ (WSX-1/TCCR) and gp $130^{5}$ that are expressed on many cell types including lymphocytes and APCs. ${ }^{6}$ Both pro- and anti-inflammatory properties of IL-27 have been reported. For example, IL-27 induces IL-12R expression, supporting subsequent Th1 differentiation. ${ }^{7}$ Mice deficient in IL-27R $\alpha$ thus exhibit elevated susceptibility to Leishmania infection, emphasizing the Th1-promoting role of IL-27. ${ }^{6}$ On the other hand, IL-27 also exhibits immunosuppressive properties. IL-27R $\alpha-/-$ mice infected with Toxoplasma gondii fail to downregulate immune responses, developing lethal T-cell-mediated immune responses. ${ }^{6}$ Particularly interesting is the immunosuppressive functions of IL-27 in the context of Th17 immunity. IL-27R $\alpha-/-$ mice are highly susceptible to the induction of Th17-mediated neuroinflammation. ${ }^{8}$ One proposed mechanism is that IL-27 induces IL-10 production by T cells. ${ }^{9}$ IL-27 also modulates regulatory T-cell (Treg) functions. IL-27 promotes the development of Treg cells that control inflammatory immunity at the site of inflammation. ${ }^{10}$ IL-27 also exacerbates inflammatory responses by restraining inducible Treg development. ${ }^{11}$

In the context of intestinal inflammation, the roles of IL-27 again remain controversial. Immunodeficient hosts transferred with IL-27R $\alpha-/-$ CD4 T cells develop attenuated colitis, which has been attributed to increased inducible Foxp $3+$ Treg conversion. ${ }^{11}$ The fact that Il27a and Ebi3 mRNA expression is upregulated in biopsy samples of active IBD patients further supports the notion that IL-27 may have a crucial proinflammatory role. ${ }^{12}$ On the other hand, a recent genome-wide

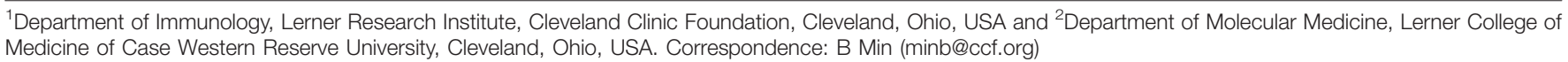

Received 17 May 2013; accepted 11 September 2013; published online 16 October 2013. doi:10.1038/mi.2013.82 
association study has identified five new regions associated with early-onset IBD susceptibility, including IL-27. ${ }^{13}$ In this study, IL-27 expression in patients with early-onset Crohn's disease was significantly lower than that in healthy control. ${ }^{13}$ In the DSS (dextran sulfate sodium) model of colitis, IL-27 can be either protective or pathogenic. ${ }^{14,15}$ With regard to IL-27 action on non-T cells, IL-27 upregulates major histocompatibility complex (MHC) and TLR4 expression in human monocytes, leading to increased production of IL- 6 and IL- $1 \beta$ upon lipopolysaccharide (LPS) stimulation in vitro. ${ }^{16,17}$ By contrast, IL-27 downregulates IL-12 production by activated macrophages in Mycobacterium tuberculosis infection model. ${ }^{18}$ The roles for IL-27 in non-T cells in vivo remain unclear.

Here, we report that IL-27 acting on APCs have a crucial role in optimizing Th17 differentiation by augmenting production of Th17-promoting cytokines. IL-27R $\alpha-/-$ lymphopenic hosts were completely protected from $\mathrm{T}$-cell-mediated colitis, whereas IL-27R $\alpha+/+$ lymphopenic mice develop fulminant inflammation in the colon. T-cell differentiation into Th17 lineage effector cells was selectively impaired in mice without IL-27R $\alpha$. APCs, primarily macrophages and dendritic cells (DCs), were defective in producing Th17-promoting cytokines, IL-1 $\beta$ and IL-6. Therefore, IL-27, acting on APCs, has an important proinflammatory function in supporting Th17 differentiation in vivo.

\section{RESULTS}

IL-27R $\alpha-I$ - Iymphopenic mice are resistant to naive CD4 T-cell-mediated colitis

Besides T cells, IL-27 also stimulates various cell types, including B cells, NK cells, macrophages, and DCs. However, its biological functions on these targets have not formally been explored. To examine whether IL-27 signaling on non-T cells affects CD4 T-cell immunity, we adoptively transferred wildtype (WT) naive CD4 T cells into TCR $\beta-/$ - recipients that express or lack IL-27R $\alpha$. We recently reported that CD4 T cells rapidly differentiate into colitogenic cells in TCR $\beta-/-$ recipients and that cells expressing IFN $\gamma$ and/or IL-17 are highly enriched in gut-associated tissues like mesenteric lymph node $(\mathrm{mLN})$ and lamina propria. ${ }^{4} \mathrm{TCR} \beta-/-$ recipients of naive CD4 T cells rapidly lost body weight (Figure 1a), and the colon tissue was heavily infiltrated with inflammatory cells (Figure 1b). Cellular infiltrates were mostly CD3 $+\mathrm{T}$ cells ${ }^{4}$ and macrophages (data not shown). By contrast, IL-27R $\alpha-/-$ TCR $\beta-/-$ recipients of CD4 $\mathrm{T}$ cells exhibited no signs of weight loss, and the colon architecture as well as goblet cells remained mostly intact with minor inflammation (Figure 1a,b). As CD4 T cells secreting IFN $\gamma$ and/or IL-17 are thought to mediate inflammatory processes, we next examined cytokine profiles of the donor T cells at 4 weeks after transfer. We first noticed that the overall expansion of donor cells in both groups was comparable (Figure 1c). However, the total number of CD4 T cells expressing intracellular IL-17A (both IL-17A + and IL-17A/IFN $\gamma+$ ) were significantly reduced in IL-27R $\alpha-/-\mathrm{TCR} \beta-/-$ recipients (Figure 1c). The failure of CD4 T cells to differentiate into Th17-type cells within IL-27R $\alpha-/$ - conditions was further supported by gene expression profiles in the $\mathrm{mLN}$. The expression of cytokines involved in Th17 differentiation, namely $I l 1 b$ and $I l 6$, was markedly decreased in IL- $27 \mathrm{R} \alpha-/-\mathrm{TCR} \beta-/-$ mice (Figure 1d). Expression of IL-12 subunits, Il12a and Il12b, was similar between the two groups (not shown). Importantly, phenotypes in IL-27R $\alpha-/-$ TCR $\beta-/-$ recipients was not due to altered Treg development, ${ }^{11}$ as Foxp3 expression of the donor T cells in both recipients was similar (not shown). Given the fact that donor T cells express the functional IL-27R, these
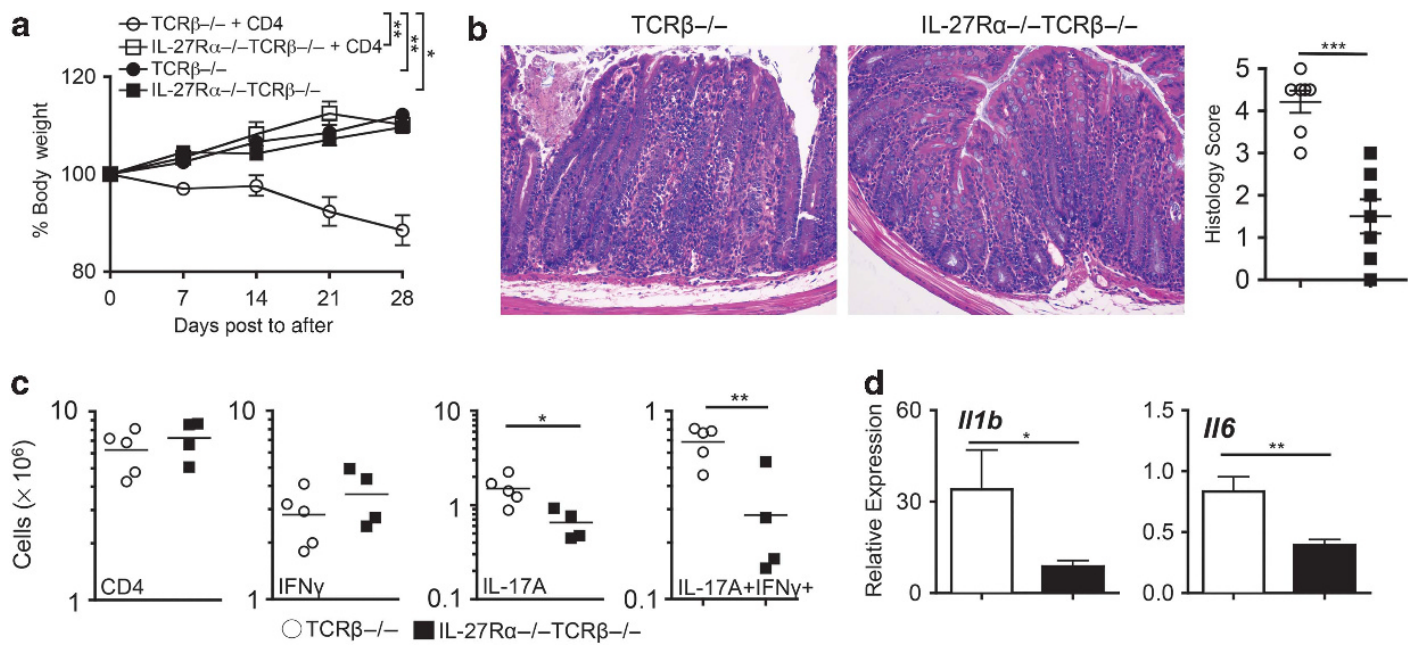

Figure 1 Mice deficient in interleukin (IL)-27R $\alpha$ fail to develop T-cell-mediated colitis. A total of $2.5 \times 10^{5}$ naive CD4 T cells were transferred into TCR $\beta-/$ - or IL-27R $\alpha-/$ - TCR $\beta-/$ - mice. (a) Weight loss was monitored weekly. (b-d) All data are from 4 weeks after transfer. (b) Hematoxylin and eosin $(\mathrm{H} \& \mathrm{E})$ staining $(\times 20)$ of the colon and colitis score. (c) Donor CD4 T-cell recovery and cytokine production after phorbol 12-myristate 13-acetate (PMA)/ionomycin stimulation from the mesenteric lymph node $(\mathrm{mLN})$. (d) Gene expression from the $\mathrm{mLN}$ tissue. All values were normalized to glyceraldehyde 3-phosphate dehydrogenase (GAPDH) expression. Data shown are from two independent experiments, $N=7-9$. Error bars indicate mean \pm s.e.m. ${ }^{\star} P<0.05 ;{ }^{\star \star} P<0.01 ;{ }^{\star \star \star} P<0.001$. IFN $\gamma$, interferon $\gamma$; TCR, T-cell receptor. 
results strongly suggest that IL-27 acting on the resident cells appears to have a central role in promoting CD4 T-cell differentiation into Th17-type effector cells and the subsequent development of colitis.

\section{CD4 T cells fail to differentiate into Th17 CD4 T cells in IL-27R $\alpha-I$ - recipients}

We next examined CD4 T-cell differentiation profiles 7 days after transfer, before the onset of colitis. IL-17A-producing cells were predominantly found in the $\mathrm{mLN}$ (Figure 2a), and $\sim 70 \%$ of them coexpressed IFN $\gamma$ in TCR $\beta-/-$ recipients (data not shown). In contrast, in IL-27R $\alpha-/-\mathrm{TCR} \beta-/$ - recipients CD4 T cells primarily became IFN $\gamma$-producing cells and Th17 differentiation was selectively impaired (Figure 2a). CD4 T-cell expansion in IL-27R $\alpha-/-\mathrm{TCR} \beta-/-$ recipients was
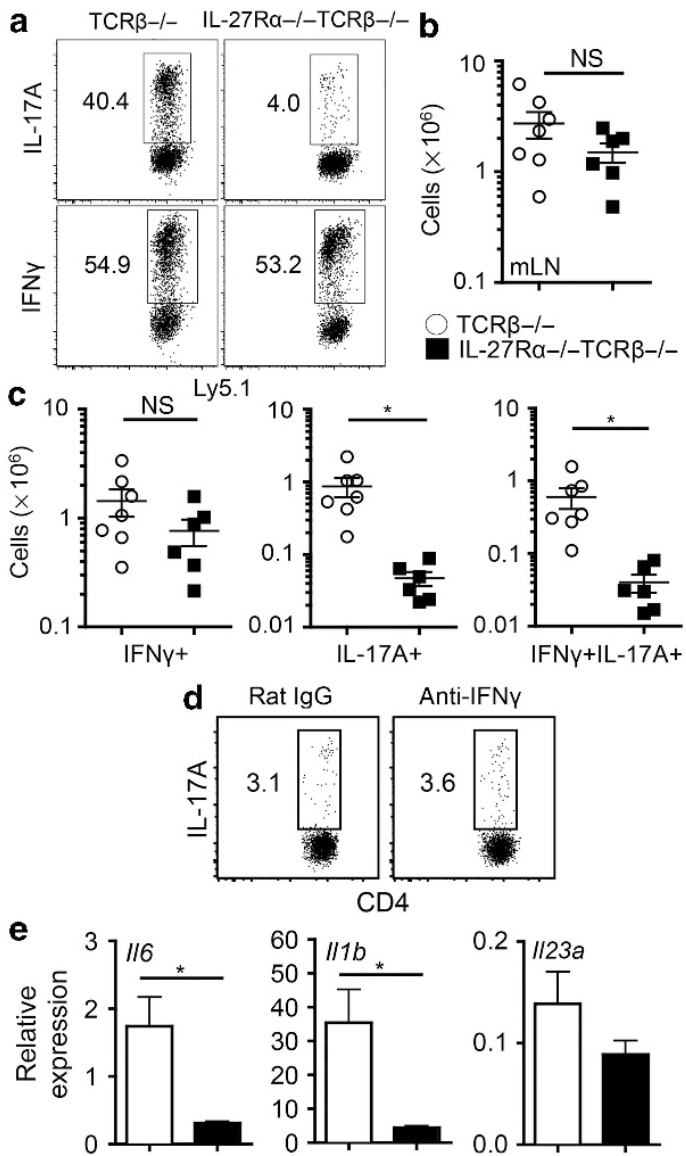

Figure 2 CD4 T cells transferred into lymphopenic TCR $\beta-/$ mice deficient in interleukin (IL)-27R $\alpha$ fail to differentiate into IL-17producing CD4 T cells. A total of $2.5 \times 10^{5}$ naive CD4 T cells were transferred into TCR $\beta-$ / - or IL-27R $\alpha-/$ - TCR $\beta-/$ - mice. All data are from 7 days after transfer. (a) Frequency of the donor CD4 T-cell cytokine production after phorbol 12-myristate 13-acetate (PMA)/ionomycin stimulation from the mesenteric lymph node $(\mathrm{mLN})$. (b) Donor cell recovery from the $\mathrm{mLN}$. (c) Number of cytokine-producing donor CD4 from the $\mathrm{mLN}$. (d) T-cell differentiation profiles in IL-27R $\alpha-/-\mathrm{TCR} \beta-/$ recipients after anti-interferon $\gamma$ (IFN $\gamma$ ) monoclonal antibody (mAb) treatment. (e) Gene expression from the $\mathrm{mLN}$ tissue. All values were normalized to glyceraldehyde 3-phosphate dehydrogenase (GAPDH) expression. Data shown are representatives of two to three independent experiments, $N=3-6$. Error bars indicate mean \pm s.e.m. ${ }^{*} P<0.05$. NS, non-significant; TCR, T-cell receptor. comparable to that in TCR $\beta-/-$ mice (Figure 2b). The total number of differentiated IL-17A-producing cells (both IL$17 \mathrm{~A}+$ and IL-17A/IFN $\gamma+$ ) were significantly reduced in IL$27 \mathrm{R} \alpha-/-\mathrm{TCR} \beta-/-$ recipients (Figure $2 \mathrm{c}$ ), whereas the absolute numbers of IFN $\gamma+$ cells remained unchanged (Figure 2c). Similar defects in Th17 differentiation was observed when neutralizing anti-IFN $\gamma$ antibody $(\mathrm{Ab})$ was injected into IL-27R $\alpha-/-$ TCR $\beta-/$ - recipients of T cells, suggesting that the impaired Th17 differentiation was not due to overproduction of IFN $\gamma$ (Figure 2d). We also found that expression of $I l 6$ and $I l 1 b$ was markedly decreased in the absence of IL-27 signaling (Figure 2e), further supporting the lack of Th17 differentiation. The expression of IL-23 was similar between the groups, suggesting that impaired Th17 differentiation was not due to differential expression of $I L-23$ (Figure 2e). Il4 expression was not found (data not shown). Il10 expression was similar between the groups; therefore, defective Th17 differentiation in IL-27R $\alpha-/$ - TCR $\beta-/$ - recipients was not due to elevated production of anti-inflammatory cytokines such as IL-10 (not shown). Collectively, these results demonstrate that the IL-27R $\alpha$ deficiency in recipient-derived cells have a key role particularly in Th17 differentiation possibly by controlling the production of Th17-promoting cytokines.

Noncolitogenic cells generated in IL-27R $\alpha-$ I- TCR $\beta-$ Irecipients still express gut-homing molecules and gut antigen specificity

The failure of colitis induction in IL-27R $\alpha-/-$ TCR $\beta-/-$ recipients may be due to defects in migration of colitogenic

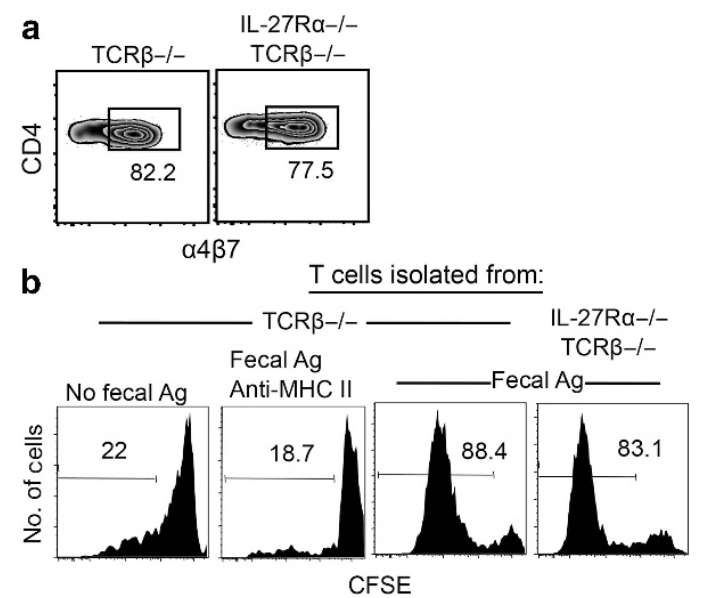

Figure 3 Failure of colitis development in interleukin (IL)-27R $\alpha-/$ TCR $\beta-/$ - recipients is not because of defects in gut homing or antigen specificity. A total of $2.5 \times 10^{5}$ naive CD4 T cells were transferred into TCR $\beta-$ / - or IL-27R $\alpha-$ / - TCR $\beta-$ / - mice. (a) $\alpha 4 \beta 7$ Expression on transferred CD4 T cells from the mesenteric lymph node $(\mathrm{mLN}) 7$ days after CD4 transfer. (b) $\alpha 4 \beta 7+$ Donor T cells were isolated from the $\mathrm{mLN}$ of TCR $\beta-$ / - or IL-27R $\alpha-$ / - TCR $\beta-/$ - recipients 7 days after transfer, carboxyfluorescein succinimidyl ester (CFSE) labeled, and cocultured with T-depleted splenocytes pulsed with fecal extract. CFSE profile of CD4 T cells was determined after a 5-day culture. In some conditions, anti-major histocompatibility complex II (MHCII)-blocking monoclonal antibody ( $\mathrm{mAb}$ ) was added. The experiments were repeated more than two times with similar results. TCR, T-cell receptor. 
T cells to the gut. To address this question, WT CD4 T cells were transferred into TCR $\beta-/$ - or IL-27R $\alpha-/$ - TCR $\beta-/$ mice and gut homing integrin, $\alpha 4 \beta 7$, expression was assessed from T cells in the mLN. mLN T cells of both recipients had no defects in upregulating $\alpha 4 \beta 7$ expression (Figure 3a). Alternatively, gut antigen (Ag) -specific colitogenic T-cell generation may be impaired in IL-27R $\alpha-/-$ TCR $\beta-/-$ mice. Donor $\mathrm{T}$ cells were isolated from the mLN and stimulated in vitro with fecal extract Ag. As shown in Figure 3b, T cells from both recipients proliferate in response to gut Ag stimulation. The proliferation did not occur without $\mathrm{Ag}$ and was completely blocked by anti-MHCII Ab (Figure 3b). Although T cells activated within IL-27R $\alpha-/-$ TCR $\beta-/$ - mice became $\alpha 4 \beta 7+-$, IFN $\gamma+-$ and gut Ag-specific cells, they are unable to induce intestinal inflammation, suggesting that IL-17 production by $\mathrm{CD} 4 \mathrm{~T}$ cells is the major contributing factor to gut inflammation. Consistent with this finding, transfer of naive CD4 T cells deficient in ROR $\gamma t$ or blockade of both IL-17A and IL-17F ameliorates intestinal inflammation. ${ }^{19,20}$

DCs and macrophages both express and respond to IL-27 To understand mechanisms underlying IL-27-mediated Th17 differentiation, it is important to examine the sources and targets of IL-27. DCs $(\mathrm{CD} 11 \mathrm{c}+)$, macrophages $(\mathrm{CD} 11 \mathrm{~b}+$ $\mathrm{CD} 11 \mathrm{c}-)$, and $\mathrm{B}$ cells $(\mathrm{CD} 19+)$ were sorted from the mLN of TCR $\beta-I-$ mice before or 7 days after T-cell transfer. Both DCs and macrophages upregulated Il27a subunit expression following T-cell transfer, whereas its expression in B cells remained undetectable (Figure 4a). Interestingly, the upregulation following $\mathrm{T}$-cell transfer was less pronounced in IL-27R $\alpha-/-$ TCR $\beta-/$ - recipients (Figure 4a). Therefore, it is possible that IL-27 acting on APCs may promote autocrine IL-27 production, further supporting Th17 differentiation. In contrast, Ebi3 expression remained unchanged, regardless of T-cell transfer in both types of recipients (not shown). The increase of IL-27p28 without Ebi3 may result in the formation of p28 homodimers, which has recently been shown to antagonize IL-27 functions. ${ }^{21}$ However, we believe that it is not likely since the concentration of p 28 homodimers needed for such antagonistic roles requires $>5$-fold increase in concentration over IL- $6 .{ }^{21}$ It was reported that non-activated DCs express minimal level of IL-27 receptors compared with that of $\mathrm{T}$ cells; ${ }^{22}$ however, the IL-27R $\alpha$ expression was detectable in all tested APCs in naive animals (Figure $4 \mathbf{b}$ ). Gp130 expression was found in DCs and macrophages, whereas CD19 + B cells were mostly gp130 - ${ }^{23}$ (Figure $4 \mathbf{b}$ ). Therefore, DCs and macrophages likely have the potential to produce and respond to IL-27. In support of this notion, DCs and macrophages upregulated the expression of both Illb and Il6 following T-cell transfer, whereas those cells from IL$27 \mathrm{R} \alpha-/$ - TCR $\beta-/$ - recipients failed to do so (Figure 4c). These results directly indicate that these cells may be the key targets of IL-27 in vivo and that IL-27 strongly induces the production of IL- 6 and IL-1 $\beta$ from these target cells. B-cell production of these cytokines was negligible.
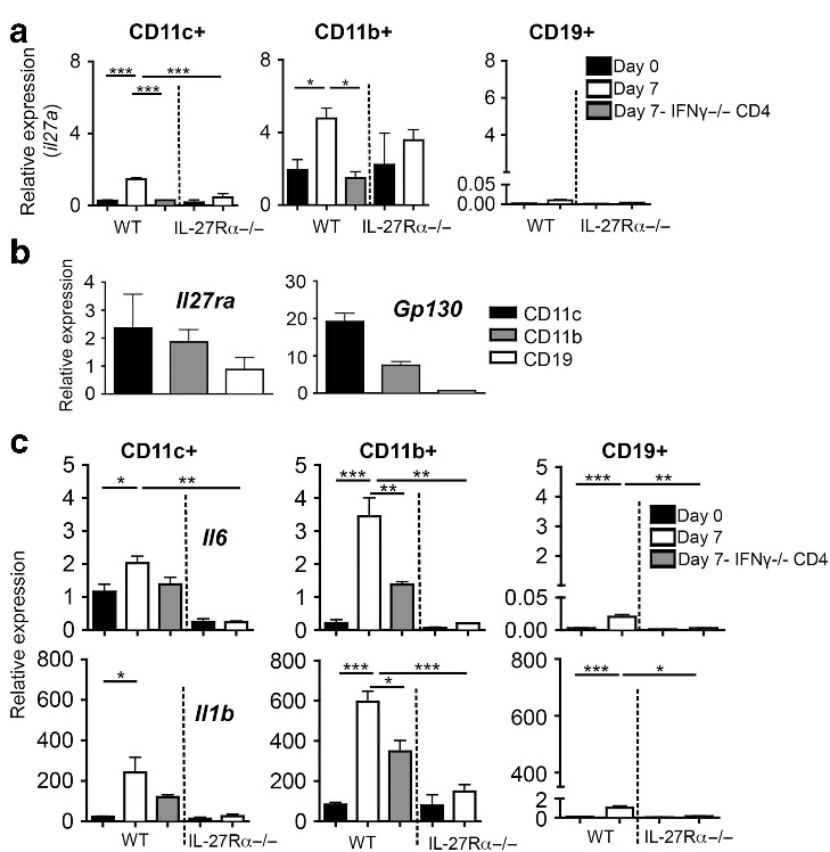

Figure 4 Dendritic cells and macrophages both express and respond to interleukin (IL)-27. (a-c) CD11c + dendritic cells, CD11b + macrophages/monocytes, and CD19 + B cells were fluorescenceactivated cell sorter (FACS) sorted from wild-type (WT) or IL-27R $\alpha-$ / mesenteric lymph node $(\mathrm{mLN})$ before or 7 days after WT or IFN $\gamma-/$ naïve CD4 transfer and examined for expression of the indicated genes. All values were normalized to the glyceraldehyde 3-phosphate dehydrogenase (GAPDH). Data shown are from two to three independent experiments $(n=5-7)$. Error bars indicate mean \pm s.e.m. ${ }^{*} P<0.05$; ${ }^{* *} P<0.01 ;{ }^{* * *} P<0.001$

IL-27 can be induced by IFNs. ${ }^{6}$ As T cells proliferating in this condition produce IFN $\gamma$, we examined if T-cell-derived IFN $\gamma$ is a key inducer of IL-27. CD4 T cells deficient in IFN $\gamma$ were transferred into TCR $\beta-1-$ mice. As shown in Figure 4a, Il27a expression in both DCs and macrophages was significantly reduced. Moreover, the expression of $I l 1 b$ and Il6 was also reduced when IFN $\gamma-/-\mathrm{CD} 4 \mathrm{~T}$ cells were transferred (Figure 4c). Of note, the reduction of Ill $b$ and Il6 in IFN $\gamma-/-$ T-cell recipients was partial compared with the reduction of IL27 expression. In support of this, transfer of IFN $\gamma-/-$ T cells into immunodeficient hosts still induce colitis (J Do and B Min, unpublished observation). ${ }^{24,25}$

\section{IL-27R $\alpha-I-$ APCs are unable to support Th17 differentiation}

We next determined if IL-27 directly enhances IL-1 $\beta$ and IL-6 from APCs. MHCII + CD19 - cells were isolated from the mLN of WT or IL-27R $\alpha-/-$ mice and stimulated with LPS overnight (Figure 5a). Although LPS stimulation of WT cells induced robust expression of $I l 1 b$ and $I l 6$ in a dose-dependent manner, IL-27R $\alpha-/-$ cell upregulation of these cytokines was impaired (Figure 5a). This finding suggests that IL-27 is produced by LPS-stimulated APCs and subsequently acts on the APCs to increase cytokine expression. To test whether the increase in proinflammatory cytokine production was directly 

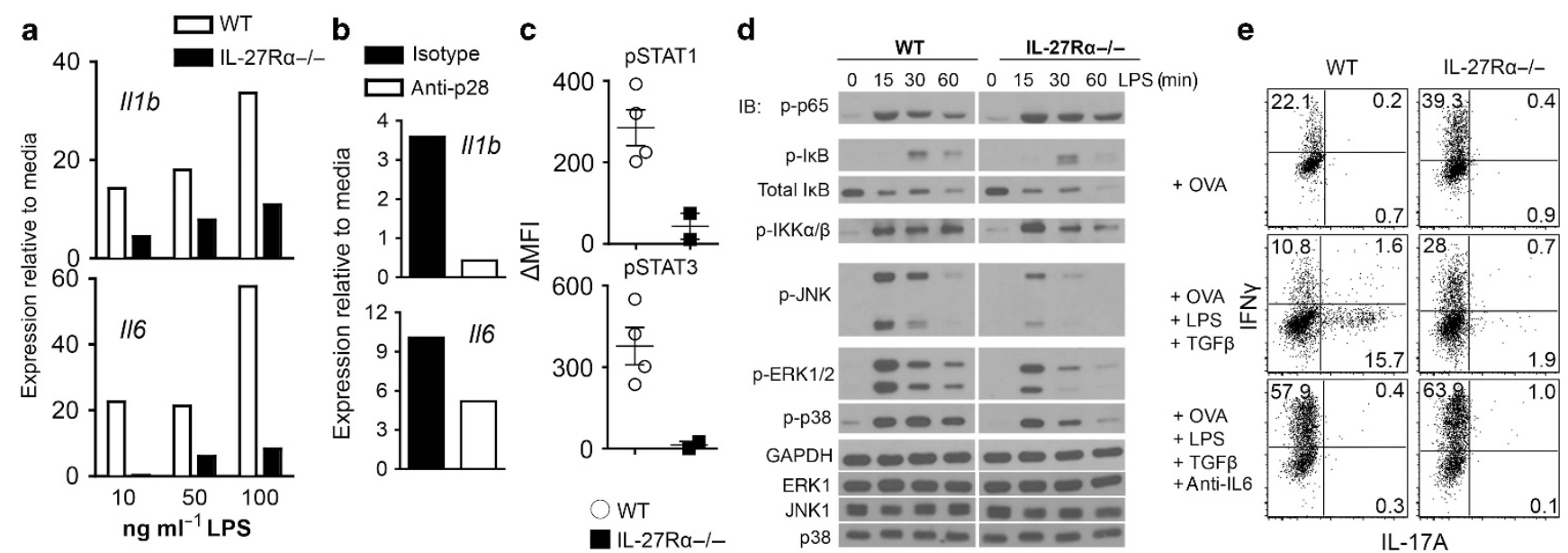

Figure 5 Interleukin (IL)-27R $\alpha-/$ - antigen-presenting cells (APCs) are unable to support T helper type 17 (Th17) differentiation. (a) Major histocompatibility complex II (MHCII) + CD19 - mesenteric lymph node (mLN) cells were fluorescence-activated cell sorter (FACS) sorted and stimulated with various concentrations of lipopolysaccharide (LPS) overnight. RNA was isolated for gene expression analysis. All values were normalized to the glyceraldehyde 3-phosphate dehydrogenase (GAPDH). (b) Wild-type (WT) peritoneal exudate cells (PECs) were treated with $10 \mathrm{ng} \mathrm{ml}^{-1}$ LPS in the presence of isotype control or anti-IL-27p28 mAb. (c) PECs were isolated from WT or IL-27R $\alpha-/-$ mice were stimulated with $10 \mathrm{ng} \mathrm{ml}^{-1} \mathrm{IL}-27$ for $15 \mathrm{~min}$ and subsequently measured for phosphorylated signal transducer and activator of transcription 1 (STAT1) and STAT3 expression. The results are expressed as delta mean fluorescence intensity ( $\triangle \mathrm{MFI})$, which is the difference between the MFI of stimulated cells and the MFI of background nonstimulated cells. (d) PECs from WT or IL-27R $\alpha-/$ - stimulated with LPS for indicated time were examined for phosphorylated protein expression by western blot analysis. (e) Naive OT-II were cocultured with PECs from WT or IL-27R $\alpha-$ / mice in the presence of exogenous tumor growth factor- $\beta$ (TGF $\beta$ ), LPS, and ovalbumin (OVA) peptide for 4 days. Cells were restimulated with phorbol 12-myristate 13-acetate (PMA)/ionomycin and cytokine expression was assessed by FACS analysis gated on OT-II cells. All data shown are representative from three to five independent experiments.

induced by IL-27, neutralizing anti-p28 Ab was added to the cultures. We found that cytokine expression was significantly diminished in the presence of anti-p28 mAb (Figure 5b). Although recent in vitro studies demonstrated that IL-27 stimulation enhances LPS-induced production of proinflammatory cytokine via TLR4 upregulation, ${ }^{17}$ we found that TLR4 expression of WT and IL-27R $\alpha-/$ - cells following T-cell transfer was similar (not shown). To examine closely molecular mechanisms underlying defective cytokine production in IL-27R $\alpha-/-$ APCs, WT and IL-27R $\alpha-/-$ peritoneal exudate cells (PECs) were harvested, stimulated with IL-27, and assessed for the intracellular signaling processes. Upon IL-27 stimulation, enhancement in pSTAT1 and pSTAT3 was seen in WT cells, whereas IL-27R $\alpha-/$ - PECs were absent in upregulating activated STAT molecules (Figure 5c), which also demonstrate that PECs do express functional IL-27R. We next assessed for activation of downstream signaling molecules following LPS stimulation. Phosphorylation of p65 and ІкB was comparable between the cell types (Figure 5d). However, the expression of phosphorylated c-Jun N-terminal kinase, extracellular signal-regulated kinase $1 / 2$, and p38 mitogen-activated protein kinase was markedly diminished in IL-27R $\alpha-/$ - PECs compared with those in WT PECs (Figure 5d). IL-27 was previously shown to regulate IL-1 $\beta$ expression in human neutrophils through p38 mitogenactivated protein kinase signaling pathways, ${ }^{26}$ suggesting that the lack of mitogen-activated protein kinase activation in IL$27 \mathrm{R} \alpha-/$ - cells may be directly related to the IL-27R $\alpha$ deficiency. Furthermore, the IL-6 promoter region contains binding elements of transcription factors including AP- $1 .{ }^{27}$ Therefore, defects in the activation of c-Jun $\mathrm{N}$-terminal kinase, extracellular signal-regulated kinase, and p38 in IL-27R $\alpha-$ / may be responsible for the failure of APCs to produce those cytokines and further to promote Th17 immunity. This notion was further supported by in vitro coculture experiments using LPS-stimulated APCs and T cells. PECs isolated from WT or IL-27R $\alpha-/$ - mice were cocultured with OT-II CD4 T cells plus ovalbumin (OVA) peptide. Addition of LPS and TGF $\beta$ without IL- 6 was sufficient to induce Th17 differentiation when WT PECs were used. However, IL-27R $\alpha-/$ - PECs were unable to support Th17 differentiation in the same condition (Figure 5e). Th17 production of OT-II cells was IL-6dependent as neutralizing IL-6 completely abolished IL-17 production by $\mathrm{CD} 4 \mathrm{~T}$ cells (Figure 5e). Therefore, these results demonstrate that IL-27 induces key cytokines involved in Th17 differentiation, mainly IL- 6 , and subsequently promotes naive T-cell differentiation into Th17 pathway.

\section{IL-27R $\alpha-I-$ CD4 T cells restore Th17 differentiation in IL-27R $\alpha-I-$ TCR $\beta-I$ - mice}

IL-27 antagonizes Th17 differentiation when it acts on T cells. ${ }^{8,9}$ As CD4 T cells transferred into lymphopenic recipients express the functional IL-27R $\alpha$, it is possible that in addition to suboptimal production of Th17-promoting cytokine production by IL-27R $\alpha-/-$ APCs, IL-27 may have an additional role on CD4 T cells, antagonizing Th17 differentiation. This 

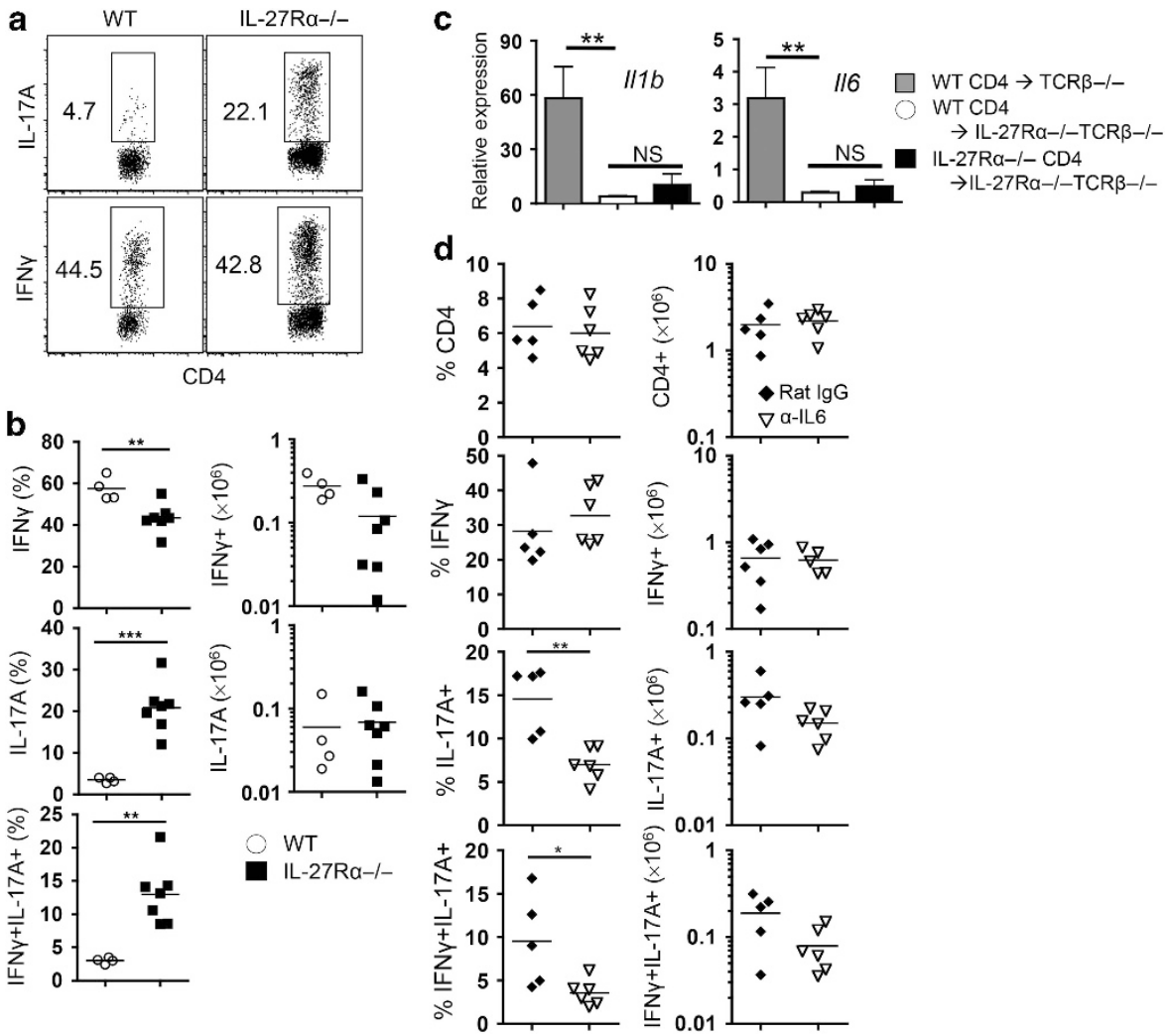

Figure 6 The lack of T helper type 17 (Th17) differentiation in interleukin (IL)-27R $\alpha-/$ - recipients is restored when T cells are unable to respond to IL-27. (a) Naive CD4 from wild-type (WT) or IL-27R $\alpha-$ / - mice were transferred into IL-27R $\alpha-$ / - TCR $\beta$ - / - recipients. Differentiation of T cells was determined 7 days after transfer. Dot plots shown represent donor T-cell intracellular cytokine expression. (b) Frequency and the absolute numbers of the cytokine-producing donor CD4 T cells in the mesenteric lymph node (mLN). (c) Gene expression from mLN tissue (day 7). All values were normalized to glyceraldehyde 3-phosphate dehydrogenase (GAPDH). (d) Naive IL-27R $\alpha-/-$ CD4 T cells were transferred into IL-27R $\alpha-/-$ TCR $\beta-/$ - recipients and given $1 \mathrm{mg}$ isotype or anti-IL-6 mAb every 3 days. The frequency and absolute numbers of the cytokine-producing CD4 T cells are shown. All data shown are representative of two to three independent experiments $(n=4-7) .{ }^{\star} P<0.05 ;{ }^{* \star} P<0.01 ;{ }^{\star \star \star \star} P<0.001$. NS, non-significant; TCR, T-cell receptor.

possibility then predicts that if $\mathrm{T}$ cells are unable to receive the antagonizing signals via IL-27, Th17 differentiation may be partially restored even in the presence of suboptimal production of Th17-promoting cytokines. Indeed, IL-27R $\alpha-/$ - naive CD4 T cells transferred into IL-27R $\alpha-/$ - TCR $\beta$ - / mice were able to differentiate into IL-17A-producing cells within IL-27R $\alpha-/-$ recipients (Figure 6a,b). Foxp3 expression in donor CD4 was slightly enhanced in mice receiving IL-27R $\alpha-/$ - compared with WT CD4 (1.7\% vs. $0.7 \%)$ but the difference did not reach a statistical significance. However, it should be noted that the total numbers of cytokineproducing $\mathrm{CD} 4 \mathrm{~T}$ cells remained low in this condition (Figure $\mathbf{6 b}$ ). The diminished expansion of IL-27R $\alpha-/-$ CD4 may be due to altered survival in the absence of IL-27 signaling. ${ }^{28}$ Likewise, tissue expression of Th17-promoting cytokines remained low compared with that in IL-27R $\alpha+/+$ conditions (Figure 6c). Low levels of IL-6 present in these conditions are likely responsible for a partial restoration of Th17 differentiation, as neutralizing IL- 6 further inhibited the development of IL-17-producing CD4 T cells (Figure 6d), whereas the overall expansion and IFN $\gamma$ production of CD4 $\mathrm{T}$ cells remained unchanged (Figure $\mathbf{6 d}$ ).

\section{IL-27-mediated Th17 differentiation also occurs in nonlymphopenic settings}

Because the experiments shown above utilized an adoptive $\mathrm{T}$-cell transfer into T-cell-deficient recipients, we wanted to confirm that IL-27-mediated Th17 differentiation is not a lymphopenia-specific finding. FACS (fluorescence-activated cell sorter) -purified naive OT-II CD4 T cells were transferred into B6 or IL-27R $\alpha-/$ - recipients, which were subsequently immunized intratracheally with OVA plus LPS. In vivo differentiation of OT-II CD4 T cells was examined 7 days after immunization. As shown in Figure 7a, a significant proportion of the transferred OT-II T cells had acquired IL-17A-producing capacity within the draining LN of B6 recipients. By contrast, IL-17A expression of OT-II CD4 $\mathrm{T}$ cells was greatly diminished in IL-27R $\alpha-/-$ recipients (Figure 7a). Impaired Th17 differentiation in IL-27R $\alpha-/-$ recipients was accompanied with elevated Th1 differentiation (Figure 7a), a finding consistent with T-cell differentiation pattern observed in lymphopenic settings (Figure 2a). Notably, the overall expansion of OT-II cells was comparable between the two groups (Figure 7b), suggesting that the defect in Th17 differentiation cannot be attributed to the lack of activation. 

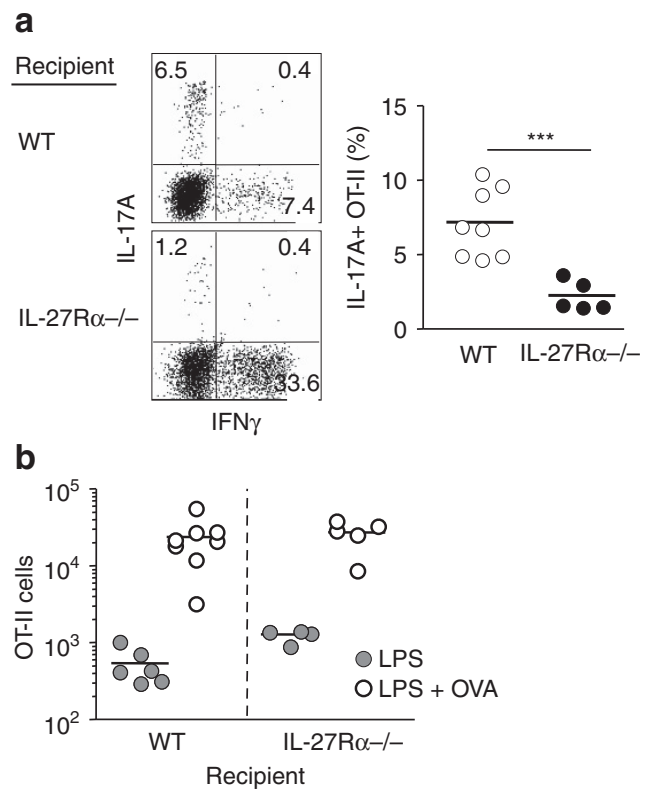

Figure 7 Thelper type 17 (Th17) differentiation is defective in interleukin (IL)-27R $\alpha-/$ - mice in nonlymphopenic models of Th17 differentiation. OT-II cells were transferred into wild-type (WT) or IL-27R $\alpha-$ / and challenged intratracheally with lipopolysaccharide (LPS) or LPS + ovalbumin (OVA). (a) Representative fluorescence-activated cell sorting (FACS) plot of donor OT-II cytokine production after LPS + OVA challenge from and (b) cell recovery 7 days after challenge from the mediastinal lymph node (LN). Data representative of two independent experiments $(n=4-8)$. ${ }^{\star \star *} P<0.001$. IFN $\gamma$, interferon $\gamma$.

These data suggest that IL-27 signaling in APCs is crucial for optimal Th17 differentiation both in lymphopenic and lymphocyte-sufficient settings.

\section{DISCUSSION}

Our study provides in vivo evidence that IL-27-mediated stimulation of non-T cells (especially APCs including macrophages and DCs) have an essential role in generating optimal Th17-type T-cell responses and intestinal inflammation. Therefore, the lack of functional IL-27R on non-T cells alone resulted in diminished production of IL- 6 and IL- $1 \beta$, leading to substantial defects in Th17 differentiation and colitis development. Importantly, impaired Th17 differentiation within IL$27 \mathrm{R} \alpha-/$ - recipients was partially reversed when $\mathrm{T}$ cells were also unable to respond to IL-27. Therefore, we would argue that when both T cells and APCs are responsive to IL-27, an optimal production of IL-1 $\beta$ and IL- 6 seems sufficient to induce a robust Th17 immunity even in the presence of IL-27-mediated action on $\mathrm{T}$ cells. With suboptimal production of these cytokines by IL-27R $\alpha-/$ - APCs, IL-27-mediated inhibitory action on $\mathrm{T}$ cells becomes more effective, inhibiting Th17 differentiation. ${ }^{9}$ When both T cells and APCs lack IL-27R, low levels of IL-1 $\beta /$ IL- 6 production by APCs are capable of inducing Th17 differentiation, although its magnitude is still substantially lower than that of optimal Th17 responses. Therefore, the balance of opposing roles of IL-27 on different target cells appears to be the key determinant of productive
Th17-type immunity. Our results also imply the key role of IL-6 (and possibly IL-1 $\beta$ ) produced by non-T cells in Th17 immunity and the pathogenesis of colitis. Consistent with this, transfer of encephalitogenic WT T cells induce less severe experimental autoimmune encephalomyelitis in IL-6-/mice, ${ }^{29}$ suggesting that APC-derived IL- 6 may be critical for the differentiation of naive T cells into Th17 lineage cells. It is still unclear how the proinflammatory roles of IL-27 dominate over its anti-Th17 function on T cells. It was recently demonstrated that IL-27 receptor mRNA expression on CD4 T cells is transiently downregulated following activation. ${ }^{22}$ This may warrant them refractory to further inhibitory signals from IL-27 during differentiation.

In the absence of IL-27R on non-T cells, it is possible that more IL-27 may be available to T cells, and this may become the dominant mechanism inhibiting Th17 differentiation. However, we would argue that suboptimal production of APCderived cytokines in response to IL-27 is the primary mechanism underlying the defects in Th17 differentiation. First, we were unable to detect increased IL-27 levels in the serum or whole colon cultures from the IL-27R $\alpha-/-$ recipients compared with WT (data not shown). Second, it was recently reported that IL-27 priming of naive T cells upregulate the expression of PD-L1, which further inhibit Th17 differentiation in trans through a PD-1-PD-L1 interaction. ${ }^{22}$ However, PD-L1 expression on WT T cells transferred into IL-27R $\alpha-/$ - recipients remained comparable to those cells transferred into IL-27R $\alpha+/+$ recipients (data not shown).

Both microbial products and cytokines such as IFNs are known to induce IL-27 production in activated APCs. ${ }^{6}$ Indeed, we observed a dramatic reduction of IL-27 expression when IFN $\gamma-/-$ CD4 T cells were transferred, indicating that IFN $\gamma$ derived from CD4 T cells may be a dominant inducer of IL-27. Nevertheless, those mice that receive IFN $\gamma-/-$ CD4 T cells still develop colitis. In support of this, we observed that the production of Th17-promoting cytokines was only partially reduced in this condition, suggesting an alternative pathway. Type I IFN is also known as a potent IL-27 inducer. ${ }^{30}$ Indeed, it was recently reported that Rag - / - mice deficient in IFNAR (human interferon a receptor) produced less IL-27, ${ }^{31}$ although these mice exhibited accelerated colitis. Severe disease seen in this condition could be attributed to low production of antiinflammatory cytokines such as IL-10 and IL-1R antagonist. ${ }^{31}$ These results suggest that IFN $\gamma$ and type I IFN, although equally potent in inducing IL-27, may contribute to the inflammation via a different mechanism.

IL-27 activates multiple pathways involving STAT1 and STAT3. ${ }^{6}$ IL-27 acts on T cells through both STAT1 to upregulate T-bet expression and STAT3 to induce IL-10 expression and inhibit inducible Treg generation. ${ }^{6}$ It is possible that in macrophages, IL-27 signals through STAT1 and STAT3, which are controlled by the expression of suppressor of cytokine signaling 3 (SOCS3). ${ }^{6}$ In CD 4 T cells, SOCS3 inhibits Th17 differentiation by inhibiting STAT3 binding to the $I l 17 a$ and Ill7f promoters and suppress IL-2 production. ${ }^{32}$ Interestingly, in monocyte/macrophage populations, macrophages 
also upregulate SOCS3 expression upon IL-27 stimulation, ${ }^{33}$ but unlike its suppressive role in CD4 T cells, SOCS3 may be essential for the development of classical/M1 inflammatory macrophages. ${ }^{34}$ Of particular interest, CD68 + macrophages in active IBD patients have increased proportion of SOCS3 + cells. ${ }^{35}$ Molecular mechanisms underlying different roles of IL-27 in different target cells are under investigation.

In the current study, we primarily focused on the role of IL-27 on APCs. However, there might be other cell population(s) besides APCs that may contribute to inflammatory responses by responding to IL-27. For example, $\gamma \delta \mathrm{T}$ cells also express IL-27 receptors and we also noticed a rapid phosphorylation of STAT1/3 following in vitro IL-27 stimulation (A Visperas and B Min, unpublished observation). Whether IL-27 stimulated $\gamma \delta$ T cells also have a role in regulating T-cell differentiation in vivo is under investigation.

It is very interesting to note that effector $\mathrm{T}$ cells generated in both conditions (i.e., Th1 type in IL-27R $\alpha-/-$ vs. Th1/Th17 type in IL-27R $\alpha+/+$ ) respond to fecal Ag stimulation. Although the precise Ag inducing this proliferation remains to be determined, these results strongly suggest that Th17 but not Th1 phenotype effector cells are essential to induce inflammatory responses leading to colitis.

In conclusion, our results demonstrate that during inflammatory responses IL-27 acts on APCs and T cells, delivering both pro- and anti-inflammatory signals, respectively. Further examination of IL-27 functions in different cell types will offer key insight into the development of Th17 immunity in vivo and may further uncover therapeutic strategies to intervene the inflammatory processes seen in Th17-associated disorders, such as multiple sclerosis and IBD.

\section{METHODS}

Mice. C57BL/6, B6 CD45.1, B6 TCR $\beta$ - / - , and B6 OT-II mice were purchased from The Jackson Laboratory (Bar Harbor, ME). B6 IL-27R $\alpha-/$ - mice were provided from Amgen (Thousand Oaks, CA). All of the mice were maintained under specific pathogen-free facility located in the Lerner Research Institute (Cleveland, OH). All animal procedures were conducted according to the guidelines of the Institutional Animal Care and Use Committee of the Cleveland Clinic.

Cell sorting and adoptive transfer. LN-naive CD4 (CD4+ $\left.\mathrm{CD} 25-\mathrm{CD} 44^{\text {low }}\right) \mathrm{T}$ cells were sorted using a FACSAria cell sorter (BD Biosciences, San Jose, CA). A total of $2.5 \times 10^{5}$ naive T cells were transferred into TCR $\beta-/-$ or IL-27R $\alpha-/-$ TCR $\beta-/-$. After $\mathrm{T}$ cells transfer, mice were weighed weekly and monitored for signs of disease. For intratracheal immunization, CD45.1 OT-II LN cells were sorted based on $\mathrm{V} \beta 5+\mathrm{CD} 4+\mathrm{CD} 25-$ and $1-1.5 \times 10^{6}$ cells were transferred one day before immunization. Some mice were also treated with $1 \mathrm{mg}$ anti-IL6 (MP5-20F3), $250 \mu \mathrm{g}$ anti-IFN $\gamma$ (XMG1.2) or rat IgG (BioXcell, West Lebanon, NH) on days 1, 2, and 5.

FACS analysis. Cells were stained with anti-CD4 (RM4-5), antiCD45.1 (A20), anti-IL-17A (17B7), anti-IFN $\gamma$ (XMG1.2), anti-Thy1.1 (HIS51), anti-CD19 (1D3), anti-CD11c (N418), anti-CD11b (M1/70), anti-Foxp3 (FJK-16a), and anti- $\alpha 4 \beta 7$ (DATK32) Abs (all Abs from eBioscience, San Diego, CA). For pSTAT staining, cells were stained with anti-STAT1 (pY701; clone 4a) and anti-STAT3 (pY705; clone 4/ P-STAT3) (from BD Biosciences, San Jose, CA). Cells were acquired using an LSRII cytometer (BD Biosciences) and analyzed using the FlowJo software (Tree Star, Ashland, OR).
Ex vivo stimulation. Tissue cells were ex vivo stimulated with phorbol 12-myristate 13 -acetate (PMA) $\left(10 \mathrm{ng} \mathrm{ml}^{-1}\right)$ and ionomycin $(1 \mu \mathrm{M})$ for $4 \mathrm{~h}$ in the presence of $2 \mu \mathrm{M}$ monensin (Calbiochem, San Diego, CA) during the last $2 \mathrm{~h}$. Cells were immediately fixed with $4 \%$ paraformaldehyde, permeabilized, and stained with fluorescenceconjugated Abs.

Real-time PCR. mLN tissue was disrupted using a TissueLyser II (Qiagen, Valencia, CA). Total RNA was extracted using an RNeasy column (Qiagen). cDNA was subsequently obtained using SuperScript III reverse transcriptase (Invitrogen, Carlsbad, CA). Real-time PCR was performed using gene-specific Taqman primer and probe sets (Applied Biosystems, Foster City, CA) and ABI 7500 PCR machine (Applied Biosystems).

Fecal Ag stimulation assay. Preparation of fecal extract and APC pulse were carried out as described previously. ${ }^{36}$ The $\alpha 4 \beta 7+$ CD 4 T cells were isolated from the mLN 7 days after transfer and labeled with carboxyfluorescein succinimidyl ester (CFSE). T cells and APCs were cultured at a 1:5 ratio (APCs pulsed with $0.3 \mathrm{mg}$ fecal extract). CFSE dilution was assessed 5 days later. In some experiments anti-MHCII $\mathrm{mAb}\left(\mathrm{Y} 3 \mathrm{P}, 10 \mu \mathrm{g} \mathrm{ml}^{-1}\right.$ ) was added.

APC stimulation. A total of $5 \times 10^{4}$ MHCII + CD19 - mLN cells were stimulated with LPS (10, 50, and $\left.100 \mathrm{ng} \mathrm{ml}^{-1}\right)$ overnight. Cells were used for qPCR. PECs were stimulated with $10 \mathrm{ng} \mathrm{ml}^{-1} \mathrm{IL}-27$ or $2 \mu \mathrm{g} \mathrm{ml}^{-1}$ LPS for intracellular signaling analysis.

In vitro Th17 differentiation. OT-II cells were stimulated with $1 \mu \mathrm{g} \mathrm{ml}^{-1}$ OVA peptide with peritoneal macrophages either stimulated with $2 \mu \mathrm{g} \mathrm{ml}^{-1}$ LPS or LPS + TGF $\beta\left(5 \mathrm{ng} \mathrm{ml}^{-1}\right)$ or LPS + TGF $\beta+$ anti-IL-6 $\left(10 \mu \mathrm{g} \mathrm{ml}^{-1}\right.$; BioXCell $)$ for 3 days. In some experiments, $20 \mu \mathrm{g} \mathrm{ml}^{-1}$ anti-IL-27 p28 mAb or goat IgG isotype control (R\&D Systems, Minneapolis, MN) was added.

Intratrachael challenge. Mice were challenged with LPS $(25 \mu \mathrm{g})$ or LPS + OVA protein $(100 \mu \mathrm{g})$ and mediastinal LN was collected 7 days after challenge for cytokine analysis.

Histology. Colon tissues were fixed in 10\% acetic acid/60\% methanol. Slides were cut and stained with hematoxylin and eosin. Colon tissues were scored in a blinded manner as reported previously ${ }^{37}$ by two individuals and scores were averaged. In brief, colon tissues were assessed at both low and high magnification to get an overall score using the scoring system: 0: no sign; 1: low infiltration and inflammation; 2: medium infiltration/inflammation; 3: high infiltration/inflammation; 4: severe infiltration with moderate loss of goblet cells and crypt structure; and 5: transmural infiltration, loss of goblet cells and crypt structure.

Western blotting. Cells stimulated as indicated were harvested, washed once with cold phosphate-buffered saline, and lysed for $30 \mathrm{~min}$ at $4{ }^{\circ} \mathrm{C}$ in $1 \%$ Triton X-100, $20 \mathrm{~mm}$ HEPES (pH 7.4), $150 \mathrm{~mm} \mathrm{NaCl}$, $12.5 \mathrm{~mm} \beta$-glycerophosphate, $1.5 \mathrm{~mm} \mathrm{MgCl}_{2}, 10 \mathrm{~mm} \mathrm{NaF}, 1 \mathrm{~mm}$ sodium orthovanadate, $2 \mathrm{~mm}$ ethylene glycol tetraacetic acid, and $1 \mathrm{~mm}$ phenylmethylsulfonyl fluoride. Cellular debris was removed by centrifugation at $10000 \mathrm{~g}$ for $15 \mathrm{~min}$. For immunoblotting, cell extracts were fractionated by sodium dodecyl sulfate-polyacrylamide gel electrophoresis and transferred to Immobilon-P transfer membranes (Millipore, Billerica, MA), using a wet transfer apparatus (Bio-Rad, Hercules, CA). Immunoblot analysis was performed, and the bands were visualized with horse radish peroxidase-coupled Ig as appropriate (Rockland, Gilbertsville, PA), using the ECL Western blotting detection system (GE Healthcare, Pittsburgh, PA). Protein levels were equilibrated with the Protein Assay Reagent (Bio-Rad).

Statistical analysis. Results represent the mean \pm s.e.m. Statistical significance was determined by the Student's $t$-test (unpaired, twotailed) or one-way analysis of variance using Prism 5 software 
(GraphPad, La Jolla, CA). A P-value $<0.05$ was considered statistically significant.

\section{ACKNOWLEDGMENTS}

We thank Ms Jennifer Powers for cell sorting, Dr Michael Freeman for scoring, and Drs Robert Fairchild, Bo Shen, Carol de la Motte, and Alex Huang for critical comments. This study was supported by NIH grants R01-Al074932 (B.M.) and T32-GM88088 (A.V.).

\section{DISCLOSURE}

The authors declared no conflict of interest.

(c) 2014 Society for Mucosal Immunology

\section{REFERENCES}

1. Zhu, J., Yamane, H. \& Paul, W.E. Differentiation of effector CD4 T cell populations (*). Annu Rev Immunol 28, 445-489 (2010).

2. Kaser, A., Zeissig, S. \& Blumberg, R.S. Inflammatory bowel disease. Annu Rev Immunol 28, 573-621 (2010).

3. Kebir, $\mathrm{H}$. et al. Preferential recruitment of interferon-gamma-expressing TH17 cells in multiple sclerosis. Ann Neurol 66, 390-402 (2009).

4. Do, J.S., Visperas, A., Dong, C., Baldwin, W.M. III \& Min, B. Cutting edge: generation of colitogenic Th17 CD4 T cells is enhanced by $\mathrm{LL}-17+$ gammadelta T cells. J Immunol 186, 4546-4550 (2011).

5. Pflanz, S. et al. WSX-1 and glycoprotein 130 constitute a signaltransducing receptor for IL-27. J Immunol 172, 2225-2231 (2004).

6. Hall, A.O., Silver, J.S. \& Hunter, C.A. The immunobiology of IL-27. Adv Immunol 115, 1-44 (2012).

7. Hibbert, L., Pflanz, S., De Waal Malefyt, R. \& Kastelein, R.A. IL-27 and IFN-alpha signal via Stat1 and Stat3 and induce T-Bet and IL-12Rbeta2 in naive T cells. J Interferon Cytokine Res 23, 513-522 (2003).

8. Batten, M. et al. Interleukin 27 limits autoimmune encephalomyelitis by suppressing the development of interleukin 17-producing $\mathrm{T}$ cells. Nat Immunol 7, 929-936 (2006).

9. Diveu, C. et al. IL-27 blocks RORc expression to inhibit lineage commitment of Th17 cells. J Immunol 182, 5748-5756 (2009).

10. Hall, A.O. et al. The cytokines interleukin 27 and interferon-gamma promote distinct Treg cell populations required to limit infection-induced pathology. Immunity 37, 511-523 (2012).

11. Cox, J.H., Kljavin, N.M., Ramamoorthi, N., Diehl, L., Batten, M. \& Ghilardi, N. IL-27 promotes T cell-dependent colitis through multiple mechanisms. J Exp Med 208, 115-123 (2011).

12. Schmidt, C. et al. Expression of interleukin-12-related cytokine transcripts in inflammatory bowel disease: elevated interleukin-23p19 and interleukin27 p28 in Crohn's disease but not in ulcerative colitis. Inflamm Bowel Dis 11, 16-23 (2005).

13. Imielinski, M. et al. Common variants at five new loci associated with earlyonset inflammatory bowel disease. Nat Genet 41, 1335-1340 (2009).

14. Honda, K. et al. Thelper 1-inducing property of IL-27/WSX-1 signaling is required for the induction of experimental colitis. Inflamm Bowel Dis 11, 1044-1052 (2005).

15. Troy, A.E. et al. IL-27 regulates homeostasis of the intestinal CD4 + effector T cell pool and limits intestinal inflammation in a murine model of colitis. $J$ Immunol 183, 2037-2044 (2009).

16. Feng, X.M. et al. Regulation of the class II and class I MHC pathways in human THP-1 monocytic cells by interleukin-27. Biochem Biophys Res Commun 367, 553-559 (2008).

17. Guzzo, C., Ayer, A., Basta, S., Banfield, B.W. \& Gee, K. IL-27 enhances LPS-induced proinflammatory cytokine production via upregulation of TLR4 expression and signaling in human monocytes. J Immunol 188, 864-873 (2012).
18. Holscher, C. et al. The IL-27 receptor chain WSX-1 differentially regulates antibacterial immunity and survival during experimental tuberculosis. J Immunol 174, 3534-3544 (2005).

19. Leppkes, M. et al. RORgamma-expressing Th17 cells induce murine chronic intestinal inflammation via redundant effects of IL-17A and IL-17F. Gastroenterology 136, 257-267 (2009).

20. Wedebye Schmidt, E.G. et al. TH17 cell induction and effects of IL-17A and IL-17F blockade in experimental colitis. Inflamm Bowel Dis 19, 1567-1576 (2013).

21. Stumhofer, J.S. et al. A role for IL-27p28 as an antagonist of gp130mediated signaling. Nat Immunol 11, 1119-1126 (2010).

22. Hirahara, K. et al. Interleukin-27 priming of $\mathrm{T}$ cells controls IL-17 production in trans via induction of the ligand PD-L1. Immunity 36, 1017-1030 (2012).

23. Oberg, H.H., Wesch, D., Grussel, S., Rose-John, S. \& Kabelitz, D. Differential expression of CD126 and CD130 mediates different STAT-3 phosphorylation in CD4 + CD25 - and CD25high regulatory T cells. Int Immunol 18, 555-563 (2006).

24. Ito, H. \& Fathman, C.G. CD45RBhigh CD4 + T cells from IFN-gamma knockout mice do not induce wasting disease. J Autoimmun 10, 455-459 (1997).

25. Simpson, S.J. et al. T cell-mediated pathology in two models of experimental colitis depends predominantly on the interleukin 12/signal transducer and activator of transcription (Stat)-4 pathway, but is not conditional on interferon gamma expression by T cells. J Exp Med 187, 1225-1234 (1998).

26. Li, J.P. et al. [IL-27 regulates the expression of Mac-1, fMLP-R and IL-1 beta in human neutrophils through p38 MAPK and PI3K signal pathways]. Zhongguo Shi Yan Xue Ye Xue Za Zhi 18, 391-395 (2010).

27. Beetz, A. et al. NF-kappaB and AP-1 are responsible for inducibility of the IL-6 promoter by ionizing radiation in HeLa cells. Int J Radiat Biol 76, 1443-1453 (2000).

28. Kim, G., Shinnakasu, R., Saris, C.J., Cheroutre, H. \& Kronenberg, M. A novel role for IL-27 in mediating the survival of activated mouse CD4 T Iymphocytes. J Immunol 2013)190, 1510-1518.

29. Mendel, I., Katz, A., Kozak, N., Ben-Nun, A. \& Revel, M. Interleukin-6 functions in autoimmune encephalomyelitis: a study in gene-targeted mice. Eur J Immunol 28, 1727-1737 (1998).

30. Molle, C., Goldman, M. \& Goriely, S. Critical role of the IFN-stimulated gene factor 3 complex in TLR-mediated IL-27p28 gene expression revealing a two-step activation process. J Immunol 184, 1784-1792 (2010).

31. Kole, A. et al. Type I IFNs regulate effector and regulatory $\mathrm{T}$ cell accumulation and anti-inflammatory cytokine production during $\mathrm{T}$ cellmediated colitis. J Immunol 191, 2771-2779 (2013).

32. Owaki, T. et al. IL-27 suppresses CD28-mediated [correction of medicated] IL-2 production through suppressor of cytokine signaling 3 . $\mathrm{J}$ Immunol 176, 2773-2780 (2006).

33. Zeitvogel, J. et al. Human primary keratinocytes show restricted ability to up-regulate suppressor of cytokine signaling (SOCS)3 protein compared with autologous macrophages. J Biol Chem 287, 9923-9930 (2012).

34. Spence, S. et al. Suppressors of cytokine signaling 2 and 3 diametrically control macrophage polarization. Immunity 38, 66-78s (2012).

35. Li, Y., de Haar, C., Peppelenbosch, M.P. \& van der Woude, C.J. SOCS3 in immune regulation of inflammatory bowel disease and inflammatory bowel disease-related cancer. Cytokine Growth Factor Rev 23, 127-138 (2012).

36. Brimnes, J., Reimann, J., Nissen, M. \& Claesson, M. Enteric bacterial antigens activate CD4 $(+)$ T cells from scid mice with inflammatory bowel disease. Eur J Immunol 31, 23-31 (2001).

37. O'Connor, W. Jr. et al. A protective function for interleukin 17A in T cellmediated intestinal inflammation. Nat Immunol 10, 603-609 (2009). 